\title{
Complementary Schur Asymptotics for Partitions
}

\author{
Jaroslav Hančl Jr.
}

December 17, 2018

\begin{abstract}
We deduce from the strong form of the Hardy-Ramanujan asymptotics for the partition function $p(n)$ an asymptotics for $p_{-S}(n)$, the number of partitions of $n$ that do not use parts from a finite set $S$ of positive integers. We apply this to construct highly oscillating partition ideals.
\end{abstract}

\section{Introduction}

Let $n \in \mathbb{N}$. We say that $\lambda=\left(\lambda_{1}, \ldots, \lambda_{k}\right)$, where $\lambda_{1} \geq \cdots \geq \lambda_{k}>0$ are positive integers, is a partition of $n$ if $\sum_{i=1}^{k} \lambda_{i}=n$. We say that $k$ is the length of $\lambda$ and $\lambda_{1}, \ldots, \lambda_{k}$ are the parts of the partition $\lambda$.

Let $p(n)$ be the number of partitions of $n$. The famous result of Hardy and Ramanujan [5] gives the asymptotics of $p(n)$ as

$$
p(n) \sim \frac{e^{C \sqrt{n}}}{4 n \sqrt{3}} \text { where } C=\pi \sqrt{2 / 3} \approx 2.565 .
$$

As usual, $f(n) \sim g(n)$ means that $\lim _{n \rightarrow \infty} \frac{f(n)}{g(n)}=1$. For more information on partitions refer to the book [1] of G. Andrews. In [6] F. Johansson presents an almost optimum numerical algorithm for evaluating $p(n)$.

One can be also interested in the number $p_{S}(n)$ of partitions of $n$ with parts from a given (finite or infinite) set $S \subset \mathbb{N}$. Such partitions are often called "restricted partitions". I. Schur [9] proved that for finite $S$ with cardinality $|S|=t$ (and such that the $\operatorname{gcd}(S)=1$ ) one has the asymptotics

$$
p_{S}(n) \sim \frac{n^{t-1}}{(t-1) ! \prod_{s \in S} s}=\frac{1}{n(t-1) !} \prod_{s \in S} \frac{n}{s} .
$$

Note that adding a new element $s$ to $S$ increases $p_{S}(n)$ by the factor $\frac{n}{s|S|}$.

Motivated by these two partition asymptotics, we obtain what we call a complementary Schur asymptotic. Nicolas and Sárközy $[7$ found an asymptotics of the number of partitions using only parts $\geq m$ for a wide range of parameter $m$. We extend this result. For a finite set of integers $S \subset \mathbb{N},|S|=t$, let $p_{-S}(n)$ 
be the number of partitions of $n$ not using any part from $S$. Our main result is that

$$
p_{-S}(n) \sim p(n)\left(\frac{C}{2 \sqrt{n}}\right)^{t} \prod_{s \in S} s=p(n) \prod_{s \in S} \frac{C s}{2 \sqrt{n}} .
$$

Now each element $s$ in $S$ decreases $p(n)$ by the factor $\frac{C s}{2 \sqrt{n}}$. We apply this to construct highly oscillating partition ideals. This theorem is an extension of result [7] of Nicolas and Sárközy who considered the case $S=[m]$.

The paper is organized as follows. In Section 2 we state the strong HardyRamanujan asymptotics of $p(n)$ and restate our main result. Section 3 presents some useful lemmas. Section 4 is devoted to the proof of the main theorem. In the last Section 5 we present an application of the main result to oscillations of growth functions of partition ideals.

\section{Two asymptotics for partitions}

Let $n \in \mathbb{N}$,

$$
\lambda_{n}:=\sqrt{n-\frac{1}{24}}, \quad C:=\pi \sqrt{\frac{2}{3}} \text { and } \quad D \in(C / 2, C)
$$

be a constant.

As we mentioned earlier, the classical asymptotics for $p(n)$ proved by Hardy and Ramanujan [5] is $p(n) \sim e^{C \sqrt{n}} / 4 n \sqrt{3}$. In [5, formula (1.55)] they gave much stronger asymptotics which we give in the next theorem. They state it as a value of a derivative which we compute explicitly.

Theorem 1 (Hardy-Ramanujan). For $n=1,2, \ldots$, the partition function $p(n)$ satisfies

$$
p(n)=\frac{e^{C \lambda_{n}}}{4 \pi \sqrt{2} \lambda_{n}^{2}}\left(C-\frac{1}{\lambda_{n}}\right)+O\left(e^{D \sqrt{n}}\right) .
$$

Note that now the error is only about a square root of the main term. This resembles strong asymptotic relations for coefficients of power series with unique dominant singularity (see P. Flajolet and R. Sedgewick [3, Chapter V] or think of the Fibonacci numbers). We use this result with small error term to deduce our asymptotic relation for $p_{-S}(n)$. Our main theorem says:

Theorem 2. Let $S=\left\{s_{1}, s_{2}, \ldots, s_{t}\right\} \subset \mathbb{N}$ be a finite set of integers with $|S|=t$. Then the number $p_{-S}(n)$ of partitions of $n$ with parts in $\mathbb{N} \backslash S$ satisfies

$$
p_{-S}(n) \sim p(n)\left(\frac{C}{2 \sqrt{n}}\right)^{t} \prod_{s \in S}=p(n) \prod_{s \in S} \frac{C s}{2 \sqrt{n}}
$$

We base our proof on manipulating the strong asymptotics of $p(n)$. First we estimate $p(n-s)$ for a fixed $s \in \mathbb{N}$, and then express $p_{-S}(n)$ as a sum of values $p(n-s)$ for various numbers $s$. 


\section{Auxiliary results}

Let $S \subset \mathbb{N}$ be a finite set with $|S|=t$ and let $s \in[t]$. First we determine the asymptotics of $p(n-s)$ and then we prove an algebraic identity needed later in the proof of the main result.

We set

$$
q(n):=\frac{e^{C \lambda_{n}}}{\lambda_{n}^{2}}\left(C-\frac{1}{\lambda_{n}}\right) .
$$

Lemma 1. Let $t \in \mathbb{N}$. Then for all $n, s \in \mathbb{N}$ with $n>s$ we have

$$
p(n-s)=\frac{e^{C \sqrt{n}}}{4 \pi n \sqrt{2}} \sum_{z=0}^{t} g(z, s) n^{-z / 2}+O\left(e^{C \sqrt{n}} n^{-\frac{t+3}{2}}\right)
$$

where for $z \in\{0,1, \ldots, t\}$ we denote by $g(z, s)$ a real polynomial in $s$ with degree $z$ and leading term

$$
g(z, s)=\frac{(-1)^{z} C^{z+1}}{2^{z} z !} s^{z}+h(z, s)
$$

where $h(z, s)$ is a real polynomial in $s$ with degree at most $z-1$.

Proof. Let $t \in \mathbb{N}$. We expand the main term in Theorem 1 in powers of $n$. Since $e^{x}=\sum_{i \geq 0} \frac{x^{i}}{i !}=\sum_{i=0}^{l} \frac{x^{i}}{i !}+O\left(x^{l+1}\right)$ for $|x|<c<1$ and $\left(c_{i}\right.$ are some real constants, not necessarily always the same)

$$
\begin{aligned}
e^{C \lambda_{n}} & =e^{C \sqrt{n}(1-1 / 24 n)^{1 / 2}}=e^{C \sqrt{n}+c_{1} n^{-1 / 2}+c_{2} n^{-2 / 2}+\cdots+c_{l} n^{-l / 2}+O\left(n^{-(l+1) / 2}\right)} \\
\frac{1}{\lambda_{n}^{2}} & =\frac{1}{n(1-1 / 24 n)}=c_{1} n^{-1}+\cdots+c_{l} n^{-l}+O\left(n^{-l-1}\right) \\
C-\frac{1}{\lambda_{n}} & =C-\frac{1}{\sqrt{n}(1-1 / 24 n)^{1 / 2}} \\
& =C+c_{1} n^{-1 / 2}+c_{2} n^{-2 / 2}+\cdots+c_{l} n^{-l / 2}+O\left(n^{-(l+1) / 2}\right)
\end{aligned}
$$

we get, for every $n>n_{0}(t)$ and some coefficients $a_{k}$,

$$
q(n)=\frac{e^{C \lambda_{n}}}{\lambda_{n}^{2}}\left(C-\frac{1}{\lambda_{n}}\right)=e^{C \sqrt{n}}\left(\sum_{k=0}^{t} a_{k} n^{-k / 2-1}+O\left(n^{-\frac{t+3}{2}}\right)\right)
$$

(note that $a_{0}=C$ ). For all integers $n>s>0$ we have, expanding again $(n-s)^{-k / 2-1}=n^{-k / 2-1}(1-s / n)^{-k / 2-1}=\ldots$ by I. Newton's binomial theorem, that

$$
\begin{aligned}
& \sum_{k=0}^{t} a_{k}(n-s)^{-k / 2-1} \\
& =\sum_{k=0}^{t} a_{k}\left(\sum_{l=0}^{t}\left(\begin{array}{c}
-1-k / 2 \\
l
\end{array}\right)(-s)^{l} n^{-1-k / 2-l}+O\left(n^{-k / 2-t-2}\right)\right) \\
& =\sum_{w=0}^{2 t} f(w, s) n^{-w / 2-1}+O\left(n^{-t-2}\right)
\end{aligned}
$$


where

$$
f(w, s)=\sum_{k, l \geq 0, k+2 l=w}(-1)^{l} a_{k}\left(\begin{array}{c}
-1-k / 2 \\
l
\end{array}\right) s^{l}
$$

is a real polynomial in $s$ with $\operatorname{deg}_{s} f(w, s)=\lfloor w / 2\rfloor$.

Next we combine expansions of the square root and of the exponential function, as above, and denote $x=n^{-1 / 2}$. For all integers $n>s>0$, where $s$ is fixed, we get

$$
\begin{aligned}
e^{C \sqrt{n-s}} & =e^{C \sqrt{n}(1-s / n)^{1 / 2}} \\
& =e^{C \sqrt{n}\left(1+\left(\begin{array}{c}
1 / 2 \\
1
\end{array}\right)(-s / n)^{1}+\cdots+\left(\begin{array}{c}
1 / 2 \\
t
\end{array}\right)(-s / n)^{t}+O\left((s / n)^{t+1}\right)\right.} \\
& =e^{C \sqrt{n}} e^{(-C / 2) s x+c_{2} s^{2} x^{3}+c_{3} s^{3} x^{5}+\cdots+c_{t} s^{t} x^{2 t-1}+O\left(s^{t+1} x^{2 t+1}\right)} \\
& =e^{C \sqrt{n}}\left(\sum_{i=0}^{t} d(i, s) n^{-i / 2}+O\left(n^{-\frac{t+1}{2}}\right)\right)
\end{aligned}
$$

where $d(i, s)$ is a polynomial in $s$ with $\operatorname{deg}_{s} d(i, s)=i$ and

$$
d(i, s)=\frac{(-1)^{i} C^{i}}{2^{i} i !} s^{i}+c_{i-1} s^{i-1}+\ldots
$$

$\left(c_{i-1} s^{i-1}+\ldots\right.$ are the remaining terms with degree less than $\left.i\right)$.

Combining results (4), (5) and (7) we have

$$
\begin{aligned}
q(n-s) & =e^{C \sqrt{n}}\left(\sum_{i=0}^{t} d(i, s) n^{-i / 2}+O\left(n^{-\frac{t+1}{2}}\right)\right)\left(\sum_{w=0}^{2 t} f(w, s) n^{-\frac{w+2}{2}}+O\left(n^{-t-2}\right)\right) \\
& =\frac{e^{C \sqrt{n}}}{n} \sum_{z=0}^{t} g(z, s) n^{-z / 2}+O\left(e^{C \sqrt{n}} n^{-\frac{t+3}{2}}\right),
\end{aligned}
$$

where

$$
g(z, s)=\sum_{i, w \geq 0, i+w=z} d(i, s) f(w, s)
$$

is a real polynomial in $s$ with $\operatorname{deg}_{s} g(z, s)=z$. Indeed, $\max _{i, w \geq 0, i+w=z}(i+$ $\lfloor w / 2\rfloor)=z$, attained uniquely for $i=z$ and $w=0$. Moreover, (6) and (8) imply

$$
g(z, s)=\frac{(-1)^{z} C^{z+1}}{2^{z} z !} s^{z}+h(z, s)
$$

where $h(z, s)$ are the remaining terms with $\operatorname{deg}_{s} h(z, s) \leq z-1$. The exponent of $C$ increased by one since $a_{0}=C$. Therefore for all integers $n>s>0$,

$$
\begin{aligned}
p(n-s) & =\frac{q(n-s)}{4 \pi \sqrt{2}}+O\left(e^{D \sqrt{n}}\right) \\
& =\frac{e^{C \sqrt{n}}}{4 \pi n \sqrt{2}} \sum_{z=0}^{t} g(z, s) n^{-z / 2}+O\left(e^{C \sqrt{n}} n^{-\frac{t+3}{2}}\right) .
\end{aligned}
$$


We state and prove an identity needed in the main proof. Recall that for $t \in \mathbb{N}_{0},[t]=\{1,2, \ldots, t\}$.

Lemma 2. Let $t \geq z \geq 0$ be integers and $s_{1}, s_{2}, \ldots, s_{t}$ be variables. Then

$$
\sum_{J \subset[t]}(-1)^{|J|}\left(\sum_{i \in J} s_{i}\right)^{z}= \begin{cases}0 & \text { for } z<t \\ (-1)^{t} t ! \prod_{i=1}^{t} s_{i} & \text { for } z=t .\end{cases}
$$

The first case holds in fact more generally for any polynomial in $\sum_{i \in J} s_{i}$ with degree at most $t-1$.

Proof. Let $k \leq t$ be positive integers, $j_{i}$ with $1 \leq j_{1}<j_{2}<\cdots<j_{k} \leq t$ be some indices and $\alpha_{1}, \ldots, \alpha_{k}$ be positive integers with $\sum \alpha_{i}=z$, so $k \leq z$. We denote the polynomial on the left side as $f=f\left(s_{1}, \ldots, s_{t}\right)$ and examine the coefficient $\left[s_{j_{1}}^{\alpha_{1}} \cdots s_{j_{k}}^{\alpha_{k}}\right] f$. Clearly, only the sets $J$ containing $\left\{j_{1}, \ldots, j_{k}\right\}$ contribute to it. Each such $J$ contributes $(-1)^{|J|}\left(\begin{array}{c}z \\ \alpha_{1}, \ldots, \alpha_{k}\end{array}\right)$ to the coefficient. Summing over all $J$ containing $\left\{j_{1}, \ldots, j_{k}\right\}$ we obtain

$$
\left[s_{j_{1}}^{\alpha_{1}} \cdots s_{j_{k}}^{\alpha_{k}}\right] f=(-1)^{k}\left(\begin{array}{c}
z \\
\alpha_{1}, \ldots, \alpha_{k}
\end{array}\right) \sum_{l=0}^{t-k}(-1)^{l}\left(\begin{array}{c}
t-k \\
l
\end{array}\right)
$$

(here $l=\left|J \backslash\left\{j_{1}, \ldots, j_{k}\right\}\right|$ ). If $z<t$ then $k<t$ and the sum is $(1-1)^{t-k}=0$ by the binomial theorem. If $z=t$ then only $k=z=t$ yields non-zero contribution, for $\alpha_{1}=\cdots=\alpha_{k}=1$, and we get the coefficient

$$
\left[s_{1} \cdots s_{t}\right] f=(-1)^{t}\left(\begin{array}{c}
z \\
1, \ldots, 1
\end{array}\right)=(-1)^{t} z !=(-1)^{t} t !
$$

which proves the theorem.

\section{Proof of the Theorem 2}

Proof of Theorem [2. Let $J \subset[t]$. Note that the partitions of $n$ using each part $s_{j}, j \in J$, at least once are in bijection with the partitions of $n-\sum_{j \in J} s_{j}$. Thus by the principle of inclusion and exclusion,

$$
p_{-S}(n)=\sum_{J \subset[t]}(-1)^{|J|} p\left(n-\sum_{j \in J} s_{j}\right) .
$$

By Lemma 1 we have

$$
\begin{aligned}
p_{-S}(n) & =\sum_{J \subset[t]}(-1)^{|J|}\left(\frac{e^{C \sqrt{n}}}{4 \pi n \sqrt{2}} \sum_{z=0}^{t} g\left(z, \sum_{j \in J} s_{j}\right) n^{-z / 2}+O\left(e^{C \sqrt{n}} n^{-\frac{t+3}{2}}\right)\right) \\
& =\frac{e^{C \sqrt{n}}}{4 \pi n \sqrt{2}} \sum_{z=0}^{t} \sum_{J \subset[t]}(-1)^{|J|} g\left(z, \sum_{j \in J} s_{j}\right) n^{-z / 2}+O\left(e^{C \sqrt{n}} n^{-\frac{t+3}{2}}\right) .
\end{aligned}
$$


We apply Lemma 2 to the polynomial $g\left(z, \sum_{j \in J} s_{j}\right)$ when $z \in[t-1]$-first we understand $s_{j}$ as variables and only at the end we substitute for them the numbers $s_{j}$-and obtain, by the first case,

$$
\sum_{J \subset[t]}(-1)^{|J|} g\left(z, \sum_{j \in J} s_{j}\right)=0 .
$$

Hence only term $z=t$ remains yielding

$$
p_{-S}(n)=\frac{e^{C \sqrt{n}}}{4 \pi n \sqrt{2}} \sum_{J \subset[t]}(-1)^{|J|} g\left(t, \sum_{j \in J} s_{j}\right) n^{-t / 2}+O\left(e^{C \sqrt{n}} n^{-\frac{t+3}{2}}\right) .
$$

Finally, we expand $g\left(t, \sum s_{j}\right)$ by equation (3) and use Lemma 2, By the first case of Lemma 2 the contributions of $h\left(z, \sum s_{j}\right)$ sum up to zero. By the second case the contribution of the leading term in $g\left(z, \sum s_{j}\right)$ is

$$
\sum_{J \subset[t]}(-1)^{|J|} \frac{(-1)^{t} C^{t+1}}{2^{t} t !}\left(\sum_{j \in J} s_{j}\right)^{t}=\frac{(-1)^{t} C^{t+1}}{2^{t} t !}(-1)^{t} t ! \prod_{i=1}^{t} s_{i}=\frac{C^{t+1}}{2^{t}} \prod_{i=1}^{t} s_{i} .
$$

Thus

$$
p_{-S}(n)=\frac{e^{C \sqrt{n}}}{4 \pi n \sqrt{2}}\left[\left(\frac{C^{t+1}}{2^{t}} \prod_{j=1}^{t} s_{j}\right) n^{-t / 2}\right]+O\left(e^{C \sqrt{n}} n^{-\frac{t+3}{2}}\right),
$$

which in view that $C=\pi \sqrt{2 / 3}$ and $p(n) \sim e^{C \sqrt{n}} / 4 n \sqrt{3}$ gives the desired asymptotics

$$
p_{-S}(n) \sim \frac{C e^{C \sqrt{n}}}{4 \pi n \sqrt{2}}\left(\frac{C}{2}\right)^{t} n^{-t / 2} \prod_{j=1}^{t} s_{j}=p(n)\left(\frac{C}{2 \sqrt{n}}\right)^{t} \prod_{j=1}^{t} s_{j} .
$$

\section{$5 \quad$ Partition functions of ideals}

Let $X$ be a set of partitions and $\lambda, \gamma \in X$. We say that $\lambda$ is a subpartition of $\gamma$ if no part from $\lambda$ has more occurrences in $\lambda$ then in $\gamma$. We denote this relation $\lambda<\gamma$. A set of partitions $X$ is a partition ideal, if $\lambda<\gamma$ and $\gamma \in X$ always implies $\lambda \in X$. By $p(n, X)$ we denote the number of partitions of $n$ lying in $X$. Let $Z$ be a set of partitions such that no two partitions in $Z$ are comparable by $<$. We denote by $F_{Z}$ the set of all partitions that do not contain any element of $Z$. Clearly, $F_{Z}$ is a partition ideal. We call $Z$ a basis of the ideal $F_{Z}$. We denote its counting function by

$$
p_{-Z}(n)=p\left(n, F_{Z}\right) .
$$


Recall that the notation $p_{-S}(n)$ and $p_{-Z}(n)$ where $S$ is a set of positive integers and $Z$ is a set of partitions. Finally, two partitions are independent if their parts are pairwise distinct.

We make use of the Cohen-Remmel theorem [2, 8, that gives sufficient condition for equality of counting functions of two partition ideals in terms of their bases. In the theorem we use the following notation. For a partition $\lambda,|\lambda|$ is the sum of all parts of $\lambda$, and for several partitions $\lambda^{i}$ their union is the partition $\lambda$ such that the multiplicity of any part equals to the maximum multiplicity attained over all $\lambda^{i}$.

Theorem 3 (Cohen, 1981; Remmel, 1982). Let $\Lambda=\left\{\lambda^{1}, \lambda^{2}, \ldots\right\}$ and $\Gamma=$ $\left\{\gamma^{1}, \gamma^{2}, \ldots\right\}$ be two finite or infinite sequences of partitions (of the same length), such that for every finite set $I \subset \mathbb{N}$,

$$
\left|\bigcup_{i \in I} \lambda^{i}\right|=\left|\bigcup_{i \in I} \gamma^{i}\right| .
$$

Then $p_{-\Lambda}(n)=p_{-\Gamma}(n)$ for every positive integer $n$.

Now we state three applications of our Theorem2, We are inspired by similar results of Hančl 4, but use a different approach.

Theorem 4. Let $F_{Z}$ be a partition ideal with basis $Z$, where $Z$ contains infinitely many pairwise independent partitions, and let $k$ be any positive integer. Then

$$
p\left(n, F_{Z}\right)<K e^{C \sqrt{n}} n^{-k}
$$

for any sufficiently large $n$, where $K=K(k, Z)$ is a fixed constant (and $C=$ $\pi \sqrt{2 / 3})$.

Proof. Let $k \in \mathbb{N}$ and $t=2 k-1$. Let $\lambda^{1}, \lambda^{2}, \ldots, \lambda^{t}$ be mutually independent partitions from $Z$ such that $\left|\lambda^{1}\right|<\left|\lambda^{2}\right|<\cdots<\left|\lambda^{t}\right|$. By Theorem 3, applied to $\Lambda=\left\{\lambda^{1}, \lambda^{2}, \ldots, \lambda^{t}\right\}$ and $\Gamma=\left\{\left(\left|\lambda^{1}\right|\right),\left(\left|\lambda^{2}\right|\right), \ldots,\left(\left|\lambda^{t}\right|\right)\right\}$,

$$
p\left(n, F_{Z}\right) \leq p_{-\Lambda}(n)=p_{-\Gamma}(n) .
$$

From Theorem 2 we have

$$
p_{-\Gamma}(n) \sim K e^{C \sqrt{n}} n^{-1-t / 2}<K e^{C \sqrt{n}} n^{-k}
$$

where $K=K(k, Z)$ is a constant and the last inequality holds for any sufficiently large $n$.

Conjecture 1. Let $X=F_{Z}$ be a partition ideal with finite basis $Z$. Then the asymptotics for the counting function $p(n, X)$ is of the form

$$
p(n, X) \sim K e^{C \sqrt{n}} n^{-1-k},
$$

where $K$ is a constant and $k=m / 2$ for some $m \in \mathbb{N}$. 
Let $\varepsilon \in(0,1)$. For the forthcoming theorem we set

$$
f(n)=\left(1-\frac{\log ^{1+\varepsilon} n}{\sqrt{n}}\right)^{2} .
$$

Thus $f(n)$ goes to 1 as fast as $n^{-1 / 2} \log ^{1+\varepsilon} n$ goes to 0 .

Theorem 5. Let $\varepsilon>0$ and $f(n)$ be as above. Then there is a partition ideal $X$ such that both

1. $p(n, X)=0$

2. $p(n, X)>p(n f(n))$

holds for infinitely many positive integers $n$.

Proof. We define the sequences $\left(s_{i}\right)_{i=1}^{\infty}$ and $\left(t_{i}\right)_{i=1}^{\infty}$ of positive integers such that $s_{1}=2$,

$$
s_{i+1}=t_{i}^{3}+2
$$

and, given $s_{i}$, we set

$$
t_{i}=\max \left\{s_{i}, \exp \left(\left(\frac{3 s_{i}+10}{2 C}\right)^{1 / \varepsilon}\right), 2 n_{0}\right\},
$$

where $n_{0}=n_{0}\left(s_{i}\right)$ is such that for any $n \geq n_{0}$ we have $f(n) \in\left(\frac{1}{2}, 1\right)$ and both

$$
2 \cdot \frac{e^{C \sqrt{n}}}{4 n \sqrt{3}}>p(n)>\frac{1}{2} \cdot \frac{e^{C \sqrt{n}}}{4 n \sqrt{3}} \quad \text { and } \quad p_{-\left[s_{i}\right]}(n)>\frac{1}{2} p(n) \prod_{s=1}^{s_{i}} \frac{C s}{2 \sqrt{n}} .
$$

Existence of $n_{0}$ follows from the Hardy-Ramanujan asymptotics (11) and Theorem 2 ,

Let $I_{i}=\left[s_{i}, t_{i}\right] \cap \mathbb{N}$. Let $X$ be the partition ideal consisting of the partitions that use parts from any of the intervals $I_{i}$ with multiplicities at most $t_{i}$, and do not use other parts. Our aim is to prove that the first condition is satisfied for $n=s_{i+1}-1$ and the second condition is satisfied for $n=t_{i+1}$.

Any partition of $s_{i+1}-1$ lying in $X$ may use only parts $\leq t_{i}$ but, as the multiplicities are restricted, sum of all the parts $\leq t_{i}$ equals

$$
\sum_{l=1}^{i} t_{l} \sum_{j=s_{l}}^{t_{l}} j \leq t_{i} \sum_{j=1}^{t_{i}} j=\frac{t_{i}^{2}\left(t_{i}+1\right)}{2}<s_{i+1}-1 .
$$

Hence easily $p\left(s_{i+1}-1, X\right)=0$ for any positive integer $i$.

Let $K=(8 \sqrt{3})^{-1}$. To prove the second property we first show that for a fixed positive integer $i$ and any $n>\max \left\{t_{i}, n_{0}\right\}$ we have

$$
p(n)^{1-\sqrt{f(n)}}>n^{3 s_{i} / 2} .
$$


Indeed, the definition of $f(n)$ implies $\sqrt{n}(1-\sqrt{f(n)})=\log ^{1+\varepsilon} n$ and $0<$ $f(n)<1$, which, combined with the definition of $t_{i}$ and $n_{0}$, yields for any $n \geq \max \left\{t_{i}, n_{0}\right\}$ bound

$$
\begin{aligned}
p(n)^{1-\sqrt{f(n)}} n^{-3 s_{i} / 2} & >K^{1-\sqrt{f(n)}} e^{C \sqrt{n}(1-\sqrt{f(n)})} n^{-1+\sqrt{f(n)}-3 s_{i} / 2} \\
& >K e^{C \log ^{1+\varepsilon} n} n^{-1-3 s_{i} / 2} \\
& =K n^{C \log ^{\varepsilon} n-1-3 s_{i} / 2} \geq K n^{4}>1,
\end{aligned}
$$

by (9) and the bounds $K>\frac{1}{16}$ and $n \geq 2$. Now Theorem 2 and (10) implies

$$
p\left(t_{i}, X\right) \geq p_{-\left[s_{i}\right]}\left(t_{i}\right)>\frac{1}{2} p\left(t_{i}\right)\left(\frac{C}{2 \sqrt{t_{i}}}\right)^{s_{i}} s_{i} !>\frac{1}{2} p\left(t_{i}\right)^{\sqrt{f\left(t_{i}\right)}}\left(\frac{C t_{i}}{2}\right)^{s_{i}} s_{i} ! .
$$

We apply again asymptotics (11) for $p\left(t_{i}\right)$ and $p\left(t_{i} f\left(t_{i}\right)\right)$ and have

$$
\begin{aligned}
p\left(t_{i}\right)^{\sqrt{f\left(t_{i}\right)}} & >\left(\frac{e^{C \sqrt{t_{i}}}}{8 \sqrt{3} t_{i}}\right)^{\sqrt{f\left(t_{i}\right)}}>\left(\frac{1}{8 \sqrt{3} t_{i}}\right)^{\sqrt{f\left(t_{i}\right)}} 2 t_{i} f\left(t_{i}\right) \sqrt{3} p\left(t_{i} f\left(t_{i}\right)\right) \\
& >\frac{1}{8}\left(8 t_{i} \sqrt{3}\right)^{1-\sqrt{f\left(t_{i}\right)}} p\left(t_{i} f\left(t_{i}\right)\right)>\frac{1}{8} p\left(t_{i} f\left(t_{i}\right)\right) .
\end{aligned}
$$

Putting these results together we get that

$$
p\left(t_{i}, X\right)>\frac{1}{16} p\left(t_{i} f\left(t_{i}\right)\right)\left(\frac{C t_{i}}{2}\right)^{s_{i}} s_{i} !>p\left(t_{i} f\left(t_{i}\right)\right)
$$

since $s_{i} \geq 2, C t_{i} \geq 6$. That completes the proof.

\section{Acknowledgments}

I would like to thank my supervisor Martin Klazar for useful conversations and corrections. The work was supported by the grant SVV-2017-260452 and SVV-2017-260456.

\section{References}

[1] G.E. Andrews. The Theory of partitions. Addison-Wesley Poblishing Co., Reading, Mass.-London-Amsterdam, 1976. Encyclopedia of Mathematics and its Applications, Vol. 2.

[2] D. I. A. Cohen. Pie-sums: a combinatorial tool for partition theory. Combin. Theory, Ser A, 31:223-236, 1981.

[3] P. Flajolet and R. Sedgewick. Analytic Combinatorics. Cambridge University Press, 2009. 
[4] J. Jr. Hančl. General enumeration of integer partitions. Diploma thesis, 2011.

[5] G. H. Hardy and S. Ramanujan. Asymptotic formulae in combinatory analysis. Proc. Lond. Math. Soc., 17:75-115, 1918

[6] F. Johansson. Efficient implementation of the Hardy-RamanujanRademacher formula. LMS J. Comput. Math., 15:341-359, 2012

[7] J. L. Nicolas and A. Sárközy. On partitions without small parts. Journal de théorie des nombres de Bordeaux, 12:227-254, 2000

[8] J. B. Remmel. Bijective proofs of some classical partition identities. J. Combin. Theory, Ser A, 33:273-286, 1982.

[9] I. Schur. Zur Additiven Zahlentheorie. S.-B. Preuss. Akad. Wiss. Phys. Math. Klasse, 488-495, 1926. 